\title{
A coupled system of fractional differential equations with nonlocal integral boundary conditions
}

\author{
Sotiris K. Ntouyas ${ }^{1}$ and Mustafa Obaid ${ }^{2 *}$
}

\section{"Correspondence:}

drmobaid@yahoo.com

${ }^{2}$ Department of Mathematics,

Faculty of Science, King Abdulaziz

University, P.O. Box 80111 , Jeddah,

21589, Saudi Arabia

Full list of author information is

available at the end of the article

\begin{abstract}
In this paper, we prove the existence and uniqueness of solutions for a system of fractional differential equations with Riemann-Liouville integral boundary conditions of different order. Our results are based on the nonlinear alternative of Leray-Schauder type and Banach's fixed-point theorem. An illustrative example is also presented.
\end{abstract} MSC: $34 \mathrm{~A} 08 ; 34 \mathrm{~A} 12 ; 34 \mathrm{~B} 15$

Keywords: Caputo fractional derivative; fractional differential systems; integral boundary conditions; fixed-point theorems

\section{Introduction}

In this paper, we investigate a boundary value problem of first-order fractional differential equations with Riemann-Liouville integral boundary conditions of different order given by

$$
\left\{\begin{array}{l}
{ }^{c} D_{0+}^{\alpha} u(t)=f(t, u(t), v(t)), \quad t \in[0,1], \\
{ }^{c} D_{0+}^{\beta} v(t)=g(t, u(t), v(t)), \quad t \in[0,1], \\
u(0)=\gamma I^{p} u(\eta)=\gamma \int_{0}^{\eta} \frac{(\eta-s)^{p-1}}{\Gamma(p)} u(s) d s, \quad 0<\eta<1, \\
v(0)=\delta I^{q} v(\zeta)=\delta \int_{0}^{\zeta} \frac{(\zeta-s)^{q-1}}{\Gamma(q)} v(s) d s, \quad 0<\zeta<1,
\end{array}\right.
$$

where ${ }^{c} D_{0+}^{\alpha},{ }^{c} D_{0+}^{\beta}$ denote the Caputo fractional derivatives, $0<\alpha, \beta \leq 1, f, g \in C([0,1] \times$ $\left.\mathbb{R}^{2}, \mathbb{R}\right)$, and $p, q, \gamma, \delta \in \mathbb{R}$.

Fractional differential equations have recently been addressed by several researchers for a variety of problems. Fractional differential equations arise in many engineering and scientific disciplines as the mathematical modeling of systems and processes in the fields of physics, chemistry, aerodynamics, electrodynamics of complex medium, polymer rheology, economics, control theory, signal and image processing, biophysics, blood flow phenomena, etc. [1-5]. Fractional-order differential equations are also regarded as a better tool for the description of hereditary properties of various materials and processes than the corresponding integer order differential equations. With this advantage, fractionalorder models become more realistic and practical than the classical integer-order models, in which such effects are not taken into account. For some recent development on the topic, see [6-18], and the references therein. The study of a coupled system of fractional

() 2012 Ntouyas and Obaid; licensee Springer. This is an Open Access article distributed under the terms of the Creative Commons Attribution License (http://creativecommons.org/licenses/by/2.0), which permits unrestricted use, distribution, and reproduction in any medium, provided the original work is properly cited. 
order is also very significant because this kind of system can often occur in applications. The reader is referred to the papers [19-22], and the references cited therein.

This paper is organized as follows: In Sect. 2, we present some basic materials needed to prove our main results. In Sect. 3, we prove the existence and uniqueness of solutions for the system (1.1) by applying some standard fixed-point principles.

\section{Preliminaries}

Let us introduce the space $X=\left\{u(t) \mid u(t) \in C^{1}([0,1])\right\}$ endowed with the norm $\|u\|=$ $\max \{|u(t)|, t \in[0,1]\}$. Obviously, $(X,\|\cdot\|)$ is a Banach space. Also, let $Y=\{v(t) \mid v(t) \in$ $\left.C^{1}([0,1])\right\}$ endowed with the norm $\|v\|=\max \{|v(t)|, t \in[0,1]\}$. The product space $(X \times$ $Y,\|(u, v)\|)$ is also a Banach space with norm $\|(u, v)\|=\|u\|+\|v\|$.

For the convenience of the readers, we now present some useful definitions and fundamental facts of fractional calculus $[1,4]$.

Definition 2.1 For at least $n$-times continuously differentiable function $g:[0, \infty) \rightarrow \mathbb{R}$, the Caputo derivative of fractional order $q$ is defined as

$$
{ }^{c} D^{q} g(t)=\frac{1}{\Gamma(n-q)} \int_{0}^{t}(t-s)^{n-q-1} g^{(n)}(s) d s, \quad n-1<q<n, n=[q]+1,
$$

where $[q]$ denotes the integer part of the real number $q$.

Definition 2.2 The Riemann-Liouville fractional integral of order $q$ is defined as

$$
I^{q} g(t)=\frac{1}{\Gamma(q)} \int_{0}^{t} \frac{g(s)}{(t-s)^{1-q}} d s, \quad q>0
$$

provided the integral exists.

The following lemmas gives some properties of Riemann-Liouville fractional integrals and Caputo fractional derivative [1].

Lemma 2.3 Let $p, q \geq 0, f \in L_{1}[a, b]$. Then $I^{p} I^{q} f(t)=I^{p+q} f(t)$ and ${ }^{c} D^{q} I^{q} f(t)=f(t)$, for all $t \in[a, b]$.

Lemma 2.4 Let $\beta>\alpha>0, f \in L_{1}[a, b]$. Then ${ }^{c} D^{\alpha} I^{\beta} f(t)=I^{\beta-\alpha} f(t)$, for all $t \in[a, b]$.

To define the solution of the boundary value problem (1.1), we need the following lemma, which deals with a linear variant of the problem (1.1).

Lemma 2.5 Let $\gamma \neq \frac{\Gamma(p+1)}{\eta^{p}}$. Then for a given $g \in C([0,1], \mathbb{R})$, the solution of the fractional differential equation

$$
{ }^{c} D^{\alpha} x(t)=g(t), \quad 0<\alpha \leq 1
$$

subject to the boundary condition

$$
x(0)=\gamma I^{p} x(\eta)=\gamma \int_{0}^{\eta} \frac{(\eta-s)^{p-1}}{\Gamma(p)} x(s) d s, \quad 0<\eta<1
$$


is given by

$$
\begin{aligned}
x(t)= & \frac{1}{\Gamma(\alpha)} \int_{0}^{t}(t-s)^{\alpha-1} g(s) d s \\
& +\frac{\gamma \Gamma(p+1)}{\Gamma(p+1)-\gamma \eta^{p}} \int_{0}^{\eta} \frac{(\eta-s)^{p+\alpha-1}}{\Gamma(p+\alpha)} g(s) d s, \quad t \in[0,1] .
\end{aligned}
$$

Proof For some constant $c_{0} \in \mathbb{R}$, we have [1]

$$
x(t)=\int_{0}^{t} \frac{(t-s)^{\alpha-1}}{\Gamma(\alpha)} g(s) d s-c_{0} .
$$

Using the Riemann-Liouville integral of order $p$ for (2.4), we have

$$
\begin{aligned}
I^{p} x(t) & =\int_{0}^{t} \frac{(t-s)^{p-1}}{\Gamma(p)}\left[\int_{0}^{s} \frac{(s-r)^{\alpha-1}}{\Gamma(\alpha)} g(r) d r-c_{0}\right] d s \\
& =I^{p} I^{\alpha} g(t)-c_{0} \frac{t^{p}}{\Gamma(p+1)}=I^{p+\alpha} g(t)-c_{0} \frac{t^{p}}{\Gamma(p+1)},
\end{aligned}
$$

where we have used Lemma 2.3. Using the condition (2.2) in the above expression, we get

$$
c_{0}=-\frac{\gamma \Gamma(p+1)}{\Gamma(p+1)-\gamma \eta^{p}} I^{p+\alpha} g(\eta)
$$

Substituting the value of $c_{0}$ in (2.4), we obtain (2.3).

\section{Main results}

For the sake of convenience, we set

$$
\begin{aligned}
& M_{1}=\frac{1}{\Gamma(\alpha+1)}+\frac{|\gamma| \eta^{p+\alpha} \Gamma(p+1)}{\Gamma(p+q+1)\left|\Gamma(p+1)-\gamma \eta^{p}\right|} \\
& M_{2}=\frac{1}{\Gamma(\beta+1)}+\frac{|\delta| \zeta^{q+\beta} \Gamma(q+1)}{\Gamma(q+\beta+1)\left|\Gamma(q+1)-\delta \zeta^{q}\right|}
\end{aligned}
$$

and

$$
M_{0}=\min \left\{1-\left(M_{1} k_{1}+M_{2} \lambda_{1}\right), 1-\left(M_{1} k_{2}+M_{2} \lambda_{2}\right)\right\} .
$$

Define the operator $T: X \times Y \rightarrow X \times Y$ by

$$
\begin{aligned}
T & (u, v)(t) \\
& =\left(\begin{array}{l}
T_{1}(u, v)(t) \\
T_{2}(u, v)(t)
\end{array}\right) \\
& =\left(\begin{array}{l}
\frac{1}{\Gamma(\alpha)} \int_{0}^{t}(t-s)^{\alpha-1} f(s, u(s), v(s)) d s+\frac{\gamma \Gamma(p+1)}{\Gamma(p+1)-\gamma \eta^{\alpha}} \int_{0}^{\eta} \frac{(\eta-s)^{p+\alpha-1}}{\Gamma(p+\alpha)} f(s, u(s), v(s)) d s \\
\frac{1}{\Gamma(\beta)} \int_{0}^{t}(t-s)^{\beta-1} g(s, u(s), v(s)) d s+\frac{\delta \Gamma(q+1)}{\Gamma(q+1)-\delta \zeta^{\beta}} \int_{0}^{\zeta} \frac{(\zeta-s)^{q+\beta-1}}{\Gamma(q+\beta)} g(s, u(s), v(s)) d s
\end{array}\right) .
\end{aligned}
$$

The first result is based on Leray-Schauder alternative. 
Lemma 3.1 (Leray-Schauder alternative, [23] p.4) Let $F: E \rightarrow E$ be a completely continuous operator (i.e., a map that restricted to any bounded set in $E$ is compact). Let

$$
\mathcal{E}(F)=\{x \in E: x=\lambda F(x) \text { for some } 0<\lambda<1\} .
$$

Then either the set $\mathcal{E}(F)$ is unbounded, or $F$ has at least one fixed point.

Theorem 3.2 Suppose that $\gamma \neq \frac{\Gamma(p+1)}{\eta^{p}}$ and $\delta \neq \frac{\Gamma(q+1)}{\zeta^{q}}$. Assume that there exist real constants $k_{i}, \lambda_{i} \geq 0(i=1,2)$ and $k_{0}>0, \lambda_{0}>0$ such that $\forall x_{i} \in \mathbb{R}(i=1,2)$, we have

$$
\begin{aligned}
& \left|f\left(t, x_{1}, x_{2}\right)\right| \leq k_{0}+k_{1}\left|x_{1}\right|+k_{2}\left|x_{2}\right|, \\
& \left|g\left(t, x_{1}, x_{2}\right)\right| \leq \lambda_{0}+\lambda_{1}\left|x_{1}\right|+\lambda_{2}\left|x_{2}\right| .
\end{aligned}
$$

In addition, it is assumed that

$$
M_{1} k_{1}+M_{2} \lambda_{1}<1 \text { and } M_{1} k_{2}+M_{2} \lambda_{2}<1
$$

where $M_{1}$ and $M_{2}$ are given by (3.1) and (3.2), respectively. Then the boundary value problem (1.1) has at least one solution.

Proof First, we show that the operator $T: X \times Y \rightarrow X \times Y$ is completely continuous. By continuity of functions $f$ and $g$, the operator $T$ is continuous.

Let $\Omega \subset X \times Y$ be bounded. Then there exist positive constants $L_{1}$ and $L_{2}$ such that

$$
|f(t, u(t), v(t))| \leq L_{1}, \quad|g(t, u(t), v(t))| \leq L_{2}, \quad \forall(u, v) \in \Omega .
$$

Then for any $(u, v) \in \Omega$, we have

$$
\begin{aligned}
\left|T_{1}(u, v)(t)\right| \leq & \frac{1}{\Gamma(\alpha)} \int_{0}^{t}(t-s)^{\alpha-1}|f(s, u(s), v(s))| d s \\
& +\frac{|\gamma| \Gamma(p+1)}{\left|\Gamma(p+1)-\gamma \eta^{p}\right|} \int_{0}^{\eta} \frac{(\eta-s)^{p+\alpha-1}}{\Gamma(p+\alpha)}|f(s, u(s), v(s))| d s \\
\leq & L_{1}\left\{\frac{1}{\Gamma(\alpha+1)}+\frac{|\gamma| \eta^{p+\alpha} \Gamma(p+1)}{\Gamma(p+q+1)\left|\Gamma(p+1)-\gamma \eta^{p}\right|}\right\}=L_{1} M_{1} .
\end{aligned}
$$

Similarly, we get

$$
\left\|T_{2}(u, v)\right\| \leq L_{2}\left\{\frac{1}{\Gamma(\beta+1)}+\frac{|\delta| \zeta^{q+\beta} \Gamma(q+1)}{\Gamma(q+\beta+1)\left|\Gamma(q+1)-\delta \zeta^{q}\right|}\right\}=L_{2} M_{2}
$$

Thus, it follows from the above inequalities that the operator $T$ is uniformly bounded.

Next, we show that $T$ is equicontinuous. Let $0 \leq t_{1} \leq t_{2} \leq 1$. Then we have

$$
\begin{aligned}
& \left|T_{1}\left(u\left(t_{2}\right), v\left(t_{2}\right)\right)-T_{1}\left(u\left(t_{1}\right), v\left(t_{1}\right)\right)\right| \\
& \quad=\left|\int_{0}^{t_{2}} \frac{\left(t_{2}-s\right)^{\alpha-1}}{\Gamma(\alpha)} f(s, u(s), v(s)) d s-\int_{0}^{t_{1}} \frac{\left(t_{1}-s\right)^{\alpha-1}}{\Gamma(\alpha)} f(s, u(s), v(s)) d s\right|
\end{aligned}
$$




$$
\begin{aligned}
& \leq \frac{L_{1}}{\Gamma(\alpha)}\left|\int_{0}^{t_{1}}\left[\left(t_{2}-s\right)^{\alpha-1}-\left(t_{1}-s\right)^{\alpha-1}\right] d s+\int_{t_{1}}^{t_{2}}\left(t_{2}-s\right)^{\alpha-1} d s\right| \\
& \leq \frac{L_{1}}{\Gamma(\alpha+1)}\left(t_{2}^{\alpha}-t_{1}^{\alpha}\right) .
\end{aligned}
$$

Analogously, we can obtain

$$
\begin{aligned}
& \left|T_{2}\left(u\left(t_{2}\right), v\left(t_{2}\right)\right)-T_{2}\left(u\left(t_{1}\right), v\left(t_{1}\right)\right)\right| \\
& \quad \leq \frac{L_{2}}{\Gamma(\beta)}\left|\int_{0}^{t_{1}}\left[\left(t_{2}-s\right)^{\beta-1}-\left(t_{1}-s\right)^{\beta-1}\right] d s+\int_{t_{1}}^{t_{2}}\left(t_{2}-s\right)^{\beta-1} d s\right| \\
& \quad \leq \frac{L_{2}}{\Gamma(\beta+1)}\left(t_{2}^{\beta}-t_{1}^{\beta}\right) .
\end{aligned}
$$

Therefore, the operator $T(u, v)$ is equicontinuous, and thus the operator $T(u, v)$ is completely continuous.

Finally, it will be verified that the set $\mathcal{E}=\{(u, v) \in X \times Y \mid(u, v)=\lambda T(u, v), 0 \leq \lambda \leq 1\}$ is bounded. Let $(u, v) \in \mathcal{E}$, then $(u, v)=\lambda T(u, v)$. For any $t \in[0,1]$, we have

$$
u(t)=\lambda T_{1}(u, v)(t), \quad v(t)=\lambda T_{2}(u, v)(t) .
$$

Then

$$
|u(t)| \leq\left\{\frac{1}{\Gamma(\alpha+1)}+\frac{|\gamma| \eta^{p+\alpha} \Gamma(p+1)}{\Gamma(p+q+1)\left|\Gamma(p+1)-\gamma \eta^{p}\right|}\right\}\left(k_{0}+k_{1}|u(t)|+k_{2}|v(t)|\right)
$$

and

$$
|v(t)| \leq\left\{\frac{1}{\Gamma(\beta+1)}+\frac{|\delta| \zeta^{q+\beta} \Gamma(q+1)}{\Gamma(q+\beta+1)\left|\Gamma(q+1)-\delta \zeta^{q}\right|}\right\}\left(\lambda_{0}+\lambda_{1}|u(t)|+\lambda_{2}|v(t)|\right) .
$$

Hence, we have

$$
\|u\| \leq M_{1}\left(k_{0}+k_{1}\|u\|+k_{2}\|v\|\right)
$$

and

$$
\|v\| \leq M_{2}\left(\lambda_{0}+\lambda_{1}\|u\|+\lambda_{2}\|v\|\right)
$$

which imply that

$$
\|u\|+\|v\|=\left(M_{1} k_{0}+M_{2} \lambda_{0}\right)+\left(M_{1} k_{1}+M_{2} \lambda_{1}\right)\|u\|+\left(M_{1} k_{2}+M_{2} \lambda_{2}\right)\|v\| .
$$

Consequently,

$$
\|(u, v)\| \leq \frac{M_{1} k_{0}+M_{2} \lambda_{0}}{M_{0}}
$$

for any $t \in[0,1]$, where $M_{0}$ is defined by (3.3), which proves that $\mathcal{E}$ is bounded. Thus, by Lemma 3.1, the operator $T$ has at least one fixed point. Hence, the boundary value problem (1.1) has at least one solution. The proof is complete. 
In the second result, we prove existence and uniqueness of solutions of the boundary value problem (1.1) via Banach's contraction principle.

Theorem 3.3 Assume that $f, g:[0,1] \times \mathbb{R}^{2} \rightarrow \mathbb{R}$ are continuous functions and there exist constants $m_{i}, n_{i}, i=1,2$ such that for all $t \in[0,1]$ and $u_{i}, v_{i} \in \mathbb{R}, i=1,2$,

$$
\left|f\left(t, u_{1}, u_{2}\right)-f\left(t, v_{1}, v_{2}\right)\right| \leq m_{1}\left|u_{1}-v_{1}\right|+m_{2}\left|u_{2}-v_{2}\right|
$$

and

$$
\left|g\left(t, u_{1}, u_{2}\right)-g\left(t, v_{1}, v_{2}\right)\right| \leq n_{1}\left|u_{1}-v_{1}\right|+n_{2}\left|u_{2}-v_{2}\right|
$$

In addition, assume that

$$
M_{1}\left(m_{1}+m_{2}\right)+M_{2}\left(n_{1}+n_{2}\right)<1,
$$

where $M_{1}$ and $M_{2}$ are given by (3.1) and (3.2), respectively. Then the boundary value problem (1.1) has a unique solution.

Proof Define $\sup _{t \in[0,1]} f(t, 0,0)=N_{1}<\infty$ and $\sup _{t \in[0,1]} g(t, 0,0)=N_{2}<\infty$ such that

$$
r \geq \frac{N_{1} M_{1}+N_{2} M_{2}}{1-M_{1}\left(m_{1}+m_{2}\right)-M_{2}\left(n_{1}+n_{2}\right)} .
$$

We show that $T B_{r} \subset B_{r}$, where $B_{r}=\{(u, v) \in X \times Y:\|(u, v)\| \leq r\}$.

For $(u, v) \in B_{r}$, we have

$$
\begin{aligned}
\left|T_{1}(u, v)(t)\right| & \\
\leq & \frac{1}{\Gamma(\alpha)} \int_{0}^{t}(t-s)^{\alpha-1}|f(s, u(s), v(s))| d s \\
& +\frac{|\gamma| \Gamma(p+1)}{\left|\Gamma(p+1)-\gamma \eta^{p}\right|} \int_{0}^{\eta} \frac{(\eta-s)^{p+\alpha-1}}{\Gamma(p+\alpha)}|f(s, u(s), v(s))| d s \\
\leq & \frac{1}{\Gamma(\alpha)} \int_{0}^{t}(t-s)^{\alpha-1}(|f(s, u(s), v(s))-f(t, 0,0)|+|f(t, 0,0)|) d s \\
& +\frac{|\gamma| \Gamma(p+1)}{\left|\Gamma(p+1)-\gamma \eta^{p}\right|} \int_{0}^{\eta} \frac{(\eta-s)^{p+\alpha-1}}{\Gamma(p+\alpha)}(|f(s, u(s), v(s))-f(t, 0,0)|+|f(t, 0,0)|) d s \\
\leq & \left\{\frac{1}{\Gamma(\alpha+1)}+\frac{|\gamma| \eta^{p+\alpha} \Gamma(p+1)}{\Gamma(p+q+1)\left|\Gamma(p+1)-\gamma \eta^{p}\right|}\right\}\left(m_{1}\|u\|+m_{2}\|v\|+N_{1}\right) \\
\leq & M_{1}\left[\left(m_{1}+m_{2}\right) r+N_{1}\right] .
\end{aligned}
$$

Hence,

$$
\left\|T_{1}(u, v)(t)\right\| \leq M_{1}\left[\left(m_{1}+m_{2}\right) r+N_{1}\right] .
$$

In the same way, we can obtain that

$$
\left\|T_{2}(u, v)(t)\right\| \leq M_{2}\left[\left(n_{1}+n_{2}\right) r+N_{2}\right] .
$$

Consequently, $\|T(u, v)(t)\| \leq r$. 
Now for $\left(u_{2}, v_{2}\right),\left(u_{1}, v_{1}\right) \in X \times Y$, and for any $t \in[0,1]$, we get

$$
\begin{aligned}
\left|T_{1}\left(u_{2}, v_{2}\right)(t)-T_{1}\left(u_{1}, v_{1}\right)(t)\right| & \\
\leq & \frac{1}{\Gamma(\alpha)} \int_{0}^{t}(t-s)^{\alpha-1}\left|f\left(s, u_{2}(s), v_{2}(s)\right)-f\left(s, u_{1}(s), v_{1}(s)\right)\right| d s \\
& +\frac{|\gamma| \Gamma(p+1)}{\left|\Gamma(p+1)-\gamma \eta^{p}\right|} \int_{0}^{\eta} \frac{(\eta-s)^{p+\alpha-1}}{\Gamma(p+\alpha)}\left|f\left(s, u_{2}(s), v_{2}(s)\right)-f\left(s, u_{1}(s), v_{1}(s)\right)\right| d s \\
\leq & \left\{\frac{1}{\Gamma(\alpha+1)}+\frac{|\gamma| \eta^{p+\alpha} \Gamma(p+1)}{\Gamma(p+q+1)\left|\Gamma(p+1)-\gamma \eta^{p}\right|}\right\}\left(m_{1}\left|u_{2}-u_{1}\right|+m_{2}\left|v_{2}-v_{1}\right|\right) \\
\leq & M_{1}\left(m_{1}\left\|u_{2}-u_{1}\right\|+m_{2}\left\|v_{2}-v_{1}\right\|\right) \\
\leq & M_{1}\left(m_{1}+m_{2}\right)\left(\left\|u_{2}-u_{1}\right\|+\left\|v_{2}-v_{1}\right\|\right),
\end{aligned}
$$

and consequently we obtain

$$
\left\|T_{1}\left(u_{2}, v_{2}\right)(t)-T_{1}\left(u_{1}, v_{1}\right)\right\| \leq M_{1}\left(m_{1}+m_{2}\right)\left(\left\|u_{2}-u_{1}\right\|+\left\|v_{2}-v_{1}\right\|\right) .
$$

Similarly,

$$
\left\|T_{2}\left(u_{2}, v_{2}\right)(t)-T_{2}\left(u_{1}, v_{1}\right)\right\| \leq M_{2}\left(n_{1}+n_{2}\right)\left(\left\|u_{2}-u_{1}\right\|+\left\|v_{2}-v_{1}\right\|\right)
$$

It follows from (3.4) and (3.5) that

$$
\left\|T\left(u_{2}, v_{2}\right)(t)-T\left(u_{1}, v_{1}\right)(t)\right\| \leq\left[M_{1}\left(m_{1}+m_{2}\right)+M_{2}\left(n_{1}+n_{2}\right)\right]\left(\left\|u_{2}-u_{1}\right\|+\left\|v_{2}-v_{1}\right\|\right) .
$$

Since $M_{1}\left(m_{1}+m_{2}\right)+M_{2}\left(n_{1}+n_{2}\right)<1$, therefore, $T$ is a contraction operator. So, by Banach's fixed-point theorem, the operator $T$ has a unique fixed point, which is the unique solution of problem (1.1). This completes the proof.

Example 3.4 Consider the following system of fractional boundary value problem:

$$
\left\{\begin{array}{l}
{ }^{c} D^{1 / 2} x(t)=\frac{1}{4(t+2)^{2}} \frac{|u(t)|}{1+|u(t)|}+1+\frac{1}{16} \sin ^{2} v(t), \quad t \in[0,1], \\
{ }^{c} D^{1 / 2} x(t)=\frac{1}{32 \pi} \sin (2 \pi u(t))+\frac{|v(t)|}{16(1+|v(t)|)}+\frac{1}{2}, \quad t \in[0,1] \\
u(0)=\sqrt{3} I^{3 / 2} u\left(\frac{1}{3}\right), \\
v(0)=\sqrt{2} I^{1 / 2} v\left(\frac{1}{2}\right) .
\end{array}\right.
$$

Here, $\alpha=1 / 2, \gamma=\sqrt{3}, p=3 / 2, \eta=1 / 3, \beta=1 / 2, \delta=\sqrt{2}, q=1 / 2, \zeta=1 / 2$, and $f(t, u, v)=$ $\frac{1}{4(t+2)^{2}} \frac{|u|}{1+|u|}+1+\frac{1}{8} \sin ^{2} v$ and $g(t, u, v)=\frac{1}{32 \pi} \sin (2 \pi u)+\frac{|v|}{16(1+|v|)}+\frac{1}{2}$. Note that $\gamma=\sqrt{3} \neq$ $\Gamma(p+1) / \eta^{p}=\Gamma(5 / 2) /(1 / 3)^{3 / 2}$ and $\delta=\sqrt{2} \neq \Gamma(q+1) / \zeta^{q}=\Gamma(3 / 2) /(1 / 2)^{1 / 2}$. Furthermore, $\left|f\left(t, u_{1}, u_{2}\right)-f\left(t, v_{1}, v_{2}\right)\right| \leq \frac{1}{16}\left|u_{1}-u_{2}\right|+\frac{1}{16}\left|v_{1}-v_{2}\right|,\left|g\left(t, u_{1}, u_{2}\right)-g\left(t, v_{1}, v_{2}\right)\right| \leq \frac{1}{16}\left|u_{1}-u_{2}\right|+$ $\frac{1}{16}\left|v_{1}-v_{2}\right|$, and

$$
\begin{aligned}
M_{1}\left(m_{1}+m_{2}\right)+M_{2}\left(n_{1}+n_{2}\right) & =\frac{1}{8}\left\{\frac{2}{\sqrt{\pi}}+\frac{\sqrt{3 \pi}}{2(9 \sqrt{\pi}-4)}\right\}+\frac{1}{16}\left\{\frac{2}{\sqrt{\pi}}+\frac{\sqrt{2 \pi}}{2(2-\sqrt{\pi})}\right\} \\
& \approx 0.712679<1 .
\end{aligned}
$$


Thus, all the conditions of Theorem 3.3 are satisfied and consequently, its conclusion applies to the problem (3.6).

\section{Competing interests}

The authors declare that they have no competing interests.

\section{Authors' contributions}

Each of the authors SKN and MO contributed to each part of this study equaly and read and approved the final version of the manuscript.

\section{Author details}

${ }^{1}$ Department of Mathematics, University of loannina, loannina, 451 10, Greece. ${ }^{2}$ Department of Mathematics, Faculty of Science, King Abdulaziz University, P.O. Box 80111, Jeddah, 21589, Saudi Arabia.

Received: 10 June 2012 Accepted: 18 July 2012 Published: 31 July 2012

\section{References}

1. Kilbas, AA, Srivastava, HM, Trujillo, Jj: Theory and Applications of Fractional Differential Equations, North-Holland Mathematics Studies, vol. 204. Elsevier, Amsterdam (2006)

2. Lakshmikantham, V, Leela, S, Vasundhara Devi, J: Theory of Fractional Dynamic Systems. Cambridge Academic Publishers, Cambridge (2009)

3. Lakshmikantham, V, Vatsala, AS: Basic theory of fractional differential equations. Nonlinear Anal. 69(8), 2677-2682 (2008)

4. Podlubny, I: Fractional Differential Equations. Academic Press, San Diego (1999)

5. Sabatier, J, Agrawal, OP, Machado, JAT (eds.): Advances in Fractional Calculus: Theoretical Developments and Applications in Physics and Engineering. Springer, Dordrecht (2007)

6. Ahmad, $B$ : Existence of solutions for irregular boundary value problems of nonlinear fractional differential equations. Appl. Math. Lett. 23, 390-394 (2010)

7. Ahmad, B, Nieto, JJ: Existence results for a coupled system of nonlinear fractional differential equations with three-point boundary conditions. Comput. Math. Appl. 58, 1838-1843 (2009)

8. Ahmad, B, Nieto, J: Existence of solutions for anti-periodic boundary value problems involving fractional differential equations via Leray-Schauder degree theory. Topol. Methods Nonlinear Anal. 35, 295-304 (2010)

9. Ahmad, B, Nieto, JJ, Pimentel, J: Some boundary value problems of fractional differential equations and inclusions. Comput. Math. Appl. 62, 1238-1250 (2011)

10. Ahmad, B, Ntouyas, SK, Alsaedi, A: New existence results for nonlinear fractional differential equations with three-point integral boundary conditions. Adv. Differ. Equ. 2011, Art. ID 107384 (2011)

11. Ahmad, B, Ntouyas, SK: A four-point nonlocal integral boundary value problem for fractional differential equations of arbitrary order. Electron. J. Qual. Theory Differ. Equ. 2011, No. 22 (2011)

12. Ahmad, B, Sivasundaram, $S$ : Existence and uniqueness results for nonlinear boundary value problems of fractional differential equations with separated boundary conditions. Commun. Appl. Anal. 13, 121-228 (2009)

13. Ahmad, B, Sivasundaram, S: On four-point nonlocal boundary value problems of nonlinear integro-differential equations of fractional order. Appl. Math. Comput. 217, 480-487 (2010)

14. Agarwal, RP, Ahmad, B: Existence theory for anti-periodic boundary value problems of fractional differential equations and inclusions. Comput. Math. Appl. 62, 1200-1214 (2011)

15. Baleanu, D, Mustafa, OG, Agarwal, RP: An existence result for a superlinear fractional differential equation. Appl. Math. Lett. 23, 1129-1132 (2010)

16. Baleanu, D, Mustafa, OG, Agarwal, RP: On $L^{p}$-solutions for a class of sequential fractional differential equations. Appl. Math. Comput. 218, 2074-2081 (2011)

17. Baleanu, D, Mustafa, OG, Agarwal, RP: Asymptotic integration of $(1+\alpha)$-order fractional differential equations Comput. Math. Appl. 62, 1492-1500 (2011)

18. Baleanu, D, Agarwal, RP, Mustafa, OG, Cosulschi, M: Asymptotic integration of some nonlinear differential equations with fractional time derivative. J. Phys. A, Math. Theor. 44(5), Article ID 055203 (2011)

19. Ahmad, B, Nieto, Jj: Existence results for a coupled system of nonlinear fractional differential equations with three-point boundary conditions. Comput. Math. Appl. 58, 1838-1843 (2009)

20. Su, X: Boundary value problem for a coupled system of nonlinear fractional differential equations. Appl. Math. Lett. $22,64-69(2009)$

21. Sun, J, Liu, Y, Liu, G: Existence of solutions for fractional differential systems with antiperiodic boundary conditions. Comput. Math. Appl. (2012). doi:10.1016/j.camwa.2011.12.083

22. Wang, J, Xiang, H, Liu, Z: Positive solution to nonzero boundary values problem for a coupled system of nonlinear fractional differential equations. Int. J. Differ. Equ. 2010, Article ID 186928 (2010)

23. Granas, A, Dugundji, J: Fixed Point Theory. Springer, New York (2005)

doi:10.1186/1687-1847-2012-130

Cite this article as: Ntouyas and Obaid: A coupled system of fractional differential equations with nonlocal integral boundary conditions. Advances in Difference Equations 2012 2012:130. 